\title{
Everything you always wanted to know about poly-L-lysine dendrigrafts (but were afraid to ask)
}

\author{
Jean-Patrick Francoia ${ }^{a}$, Laurent Vial $^{* b, c}$
}

October 18, 2017

\begin{abstract}
Less than a decade ago, dendrigrafts of poly- $L$-lysine (DGLs) joined the family of polycationic dendritic macromolecules. Resulting from the iterative polycondensation of a $\mathrm{N}$-carboxyanhydride in water, four generations of the dendrigraft can be obtained on a multi-gram scale and without chromatographic purification. DGLs share features with both dendrimers and hyperbranched polymers, but turned out to have unique biophysical and bioactive properties. The macromolecules - in their native form or functionalized - have been extensively characterized by various analytical and computational methods, and already found numerous applications in the biomedical field such as drug and gene delivery, biomaterials, tissue engineering, bioimaging, and biosensing. Despite a growing interest for DGLs, there is still plenty of room for further exciting developments that could result from a better exposure of these macromolecules, which is the ambition of this short review.
\end{abstract}

\section{Introduction}

Dendritic polymers are the fourth major class of synthetic polymer architectures (after linear, crosslinked and branched polymers). Among them, dendrimers are macromolecular objects obtained after a generational synthesis (Figure 1).[1] This route engages successive cycles of protection/deprotection or activation steps on monomeric building blocks, resulting into the formation of theoretically monodisperse structures. At the opposite of dendrimers, hyperbranched polymers are polydisperse. They are obtained by the self-condensation of unprotected building blocks, in a so-called one-pot synthesis. Starting from a building block with three branching points, one can theoretically obtain $2^{\text {n- }}$ (excluding any operation of symmetry) structures for a polymer made of $n$ monomers. Finally, dendrigrafts - the youngest member of the family of dendritic polymers - lies in between the above two.[2] The synthetic route to dendrigrafts is generational, but usually involves cycles of polymerization initiated from functional sites located on a core polymer. As a result, dendrigrafts share features with both dendrimers (i.e. generation-based growth and narrow molecular weight distribution), and hyperbranched polymers (i.e. broad number of regioisomers and rapid increase in molecular weight per generation).

\footnotetext{
${ }^{a}$ WestCHEM, School of Chemistry, The University of Glasgow, Glasgow G12 8QQ

${ }^{b}$ Institut des Biomolécules Max Mousseron, UMR 5247 CNRS - Université de Montpellier - ENSCM, Place Eugène Bataillon, 34296 Montpellier cedex 5, France

${ }^{c}$ Institut de Chimie et Biochimie Moléculaires et Supramoléculaires, UMR 5246 CNRS - Université Claude Bernard Lyon 1 - CPE Lyon - INSA, 43 Boulevard du 11 Novembre 1918, 69622 Villeurbanne cedex, France
} 

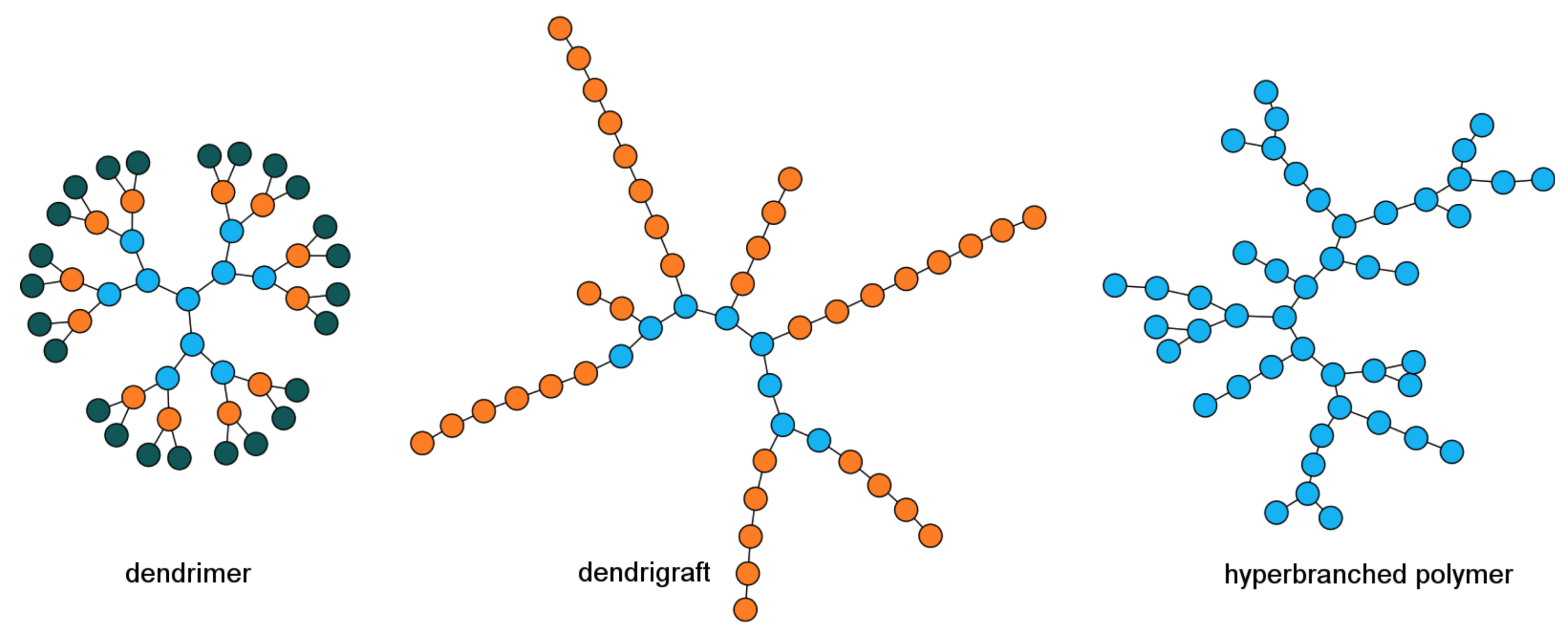

hyperbranched polymer

Figure 1: Each dot represents a monomeric building block, and each generation is highlighted with a distinct color.

Among the impressive number of dendritic polymers reported in the literature so far, the poly(amine)based ones, which are represented by the prominent poly(amidoamine) (PAMAM) and poly (L-Lysine) (DPL) dendrimers, the hyperbranched poly(ethylenimine) polymers (PEI) and poly(LLysine) (HPL) polymers, and the linear poly (L-Lysine) polymers (LPL), certainly attracted the most attention from the community of researchers working in the field of chemical biology. Historically, poly- $L$-lysine dendrons were reported for the first time in a patent by Denkewalter et al. before the advent of PAMAMs, [3, 4] and were rapidly exploited during the 1980s for the creation of synthetic vaccines. [5] The polycationic nature of these poly(amine)-based polymers at physiological $\mathrm{pH}$ is appropriate to cellular adhesion, endocytosis, and intracellular trafficking for therapeutic delivery, genetic material transfection, or imaging in cellulo. [6] However, these abiotic macromolecules exhibit some unwanted features considering their cytotoxicity and non-degradability (PAMAM and PEI), low synthetic availability (PAMAM and DPL), non-sustainable synthesis (PAMAM, DPL and PEI), high dispersities (PEI, HPL and LPL), and batch-to-batch variation (PEI and HPL), [7, 8, 9, 10] highlighting therefore the need of a new generation of dendritic polymers that overcome those major drawbacks for real-world bio-applications.[11]

This review aims at concisely presenting the innovative works reported to date in the literature on a very recent class of polycationic dendritic polymers, the poly- $L$-lysine dendrigrafts (DGLs), regarding their chemical and biological features.

\section{Chemistry}

\section{Synthesis}

Through investigations on the possible role of the N-carboxyanhydride (NCA) motif in the chemistry of the prebiotic world, Commeyras et al. discovered that its aminolysis occurs one hundred times faster than its hydrolysis in weakly acidic aqueous solutions, paving the way of a new synthetic route toward dendritic polypeptides.[12] In water at $\mathrm{pH}$ 6.5, the slow hydrolysis of the side chain-protected building block Lys(Tfa)-NCA (Figure 2) unlocks its $\alpha$-amine function that quickly react with a non-hydrolysed Lys(Tfa)-NCA molecule to form an amide bond for, after multiple similar condensation reactions, leading to the spontaneous precipitation in the reaction medium of a side chain-protected linear poly$L$-lysine. A key factor in the success of this synthetic route is certainly the decrease in solubility of 
the macromolecules as their molecular weight increases, which is due to the hydrophobicity of $\mathrm{N} \epsilon$ protected side chains. Subsequent isolation by centrifugation and aqueous alkaline deprotection afford poly- $L$-lysine $\mathbf{G 1}$. By applying the previous sequence in the presence of $\mathbf{G 1}$ as a macro-initiator, the second-generation dendrigraft G2 is obtained. It was essential to limit the Lys(Tfa)-NCA/macroinitiator ratio during this step, so that linear oligomers do not grow in the reaction medium. From this generational approach four generations of DGLs G1-G4 can be synthesised with typical yields of $50-60 \%$ on a multi-gram scale, thanks to a simple, sustainable, and scalable procedure with no batch-tobatch variability (up to the seventh-generation dendrigraft can actually be obtained using this protocol, but all the reported applications in this review only involve the first- to fourth-generation DGLs).

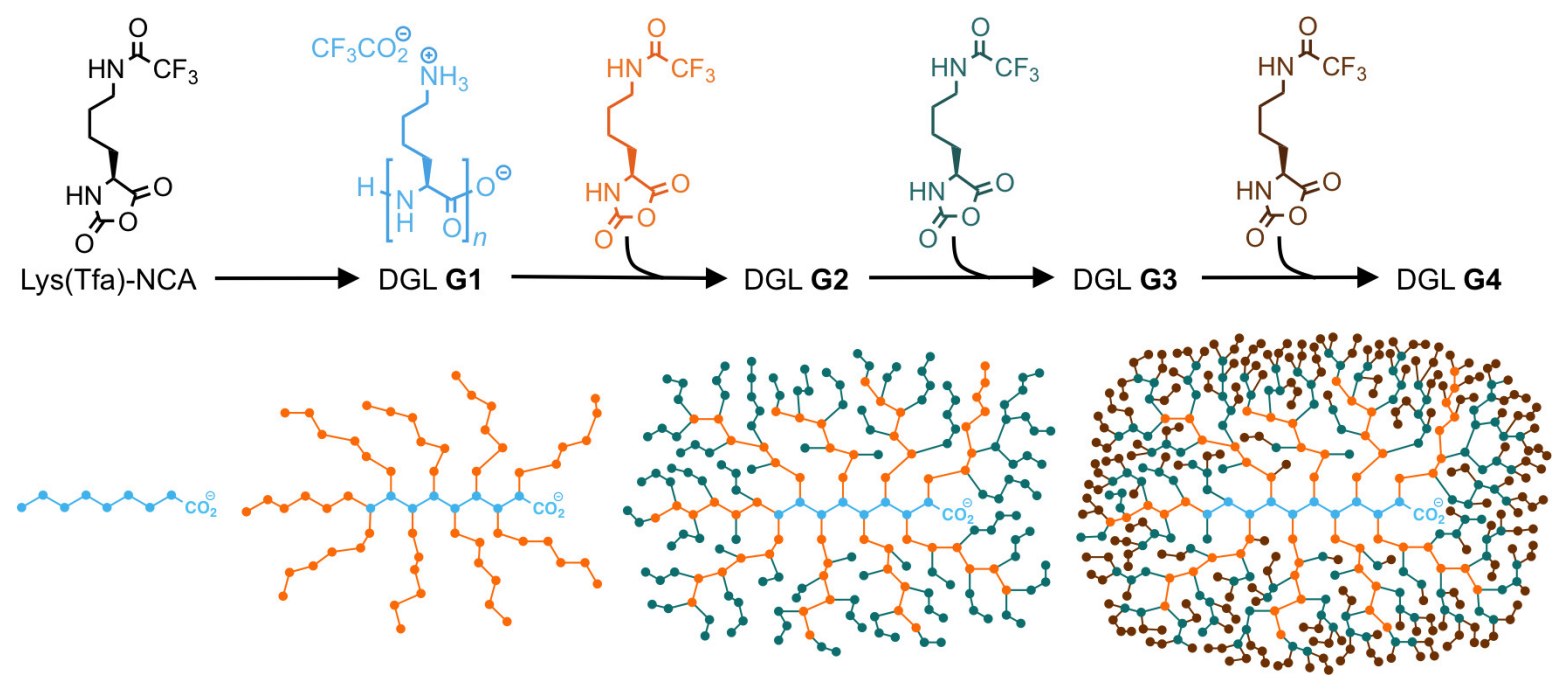

Figure 2: Synthetic route toward first- to fourth-generation DGLs G1-G4, and their respective schematic representation (each dot represents a $L$-lysine residue, pending free amino groups are not represented). The synthesis of each generation involves the following sequence: polycondensation in water, centrifugation for collecting the precipitate, alkaline deprotection of the side chains, and concentration in vacuo.

\section{Characterization}

Various analytical techniques were used to extensively characterize the poly- $L$-lysine dendrigrafts, including NMR spectrometry,[12] dynamic light scattering, Taylor dispersion analysis, size exclusion chromatography,[13] refractometry,[14] capillary isotachophoresis,[15] potentiometry,[16] colorimetry,[17] cyclic voltametry,[18] and Raman spectroscopy.[19] Table 1 gathers few selected physicochemical properties for DGLs G1-G4. It should be highlighted that such a simple synthetic procedure exerts an unexpected control on the molecular weight and diversity of the resulting dendrigrafts. Indeed, polydispersity indexes measured for DGLs (1.20-1.46) are below those of both LPLs and HPLs (1.3-2.6 and 1.8-2.6, respectively), and are comparable to those of genuine DPLs (1.1-1.5) that are only accessible via a laborious multistep process.[9] One may assume that it results from a kinetic control linked to steric hindrance between the growing chains, as well as a thermodynamic control linked to the precipitation of the protected polymers in water. Since DGLs are made of $L$-lysine residues, they could initially be assimilated to proteins. However, some of the residues are linked to each other through isopeptidic bonds (i.e. amide linkages that involve the nitrogen atom of the lateral chain of the amino acids), resulting in the simultaneous presence of free $\epsilon$ - and $\alpha$-amino groups in the biopolymers. Potentiometric titrations suggested that only the $\epsilon$-amines were protonated at physiological pH.[16] In addition, microsecond molecular dynamics simulations on DGLs G1-G4 revealed that the dendrigrafts 
Table 1: Selected physico-chemical properties of DGLs G1-G4 (with the corresponding references $[12,13,16])$.

\begin{tabular}{lccccc}
\hline & G1 & G2 & G3 & G4 & Ref. \\
\hline Molecular Weight (g.mol-1) & - & 11800 & 32100 & 88800 & {$[12]$} \\
Degree of polymerisation & 8 & 48 & 123 & 365 & {$[12]$} \\
Branching ratio (\%) & - & 12 & 24 & 23 & {$[16]$} \\
Polydispersity index & 1.20 & 1.38 & 1.46 & 1.36 & {$[12]$} \\
Hydrodynamic radius (nm) & 0.77 & 1.40 & 2.14 & 3.00 & {$[13]$} \\
Number of peptidic bonds & 7 & 41 & 92 & 280 & {$[16]$} \\
Number of isopeptidic bonds & 0 & 6 & 30 & 84 & {$[16]$} \\
Net charge at physiological pH & - & +41 & +92 & +280 & {$[16]$} \\
\hline
\end{tabular}

adopt intermediate morphologies between DPLs and LPLs, which may remind those of unfolded and intrinsically disordered proteins respectively. The simulations also highlighted their unique conformational plasticity (Figure 3), which may explain why DGLs are such efficient binders for anionic biomolecules, even in the most competitive media (vide infra).

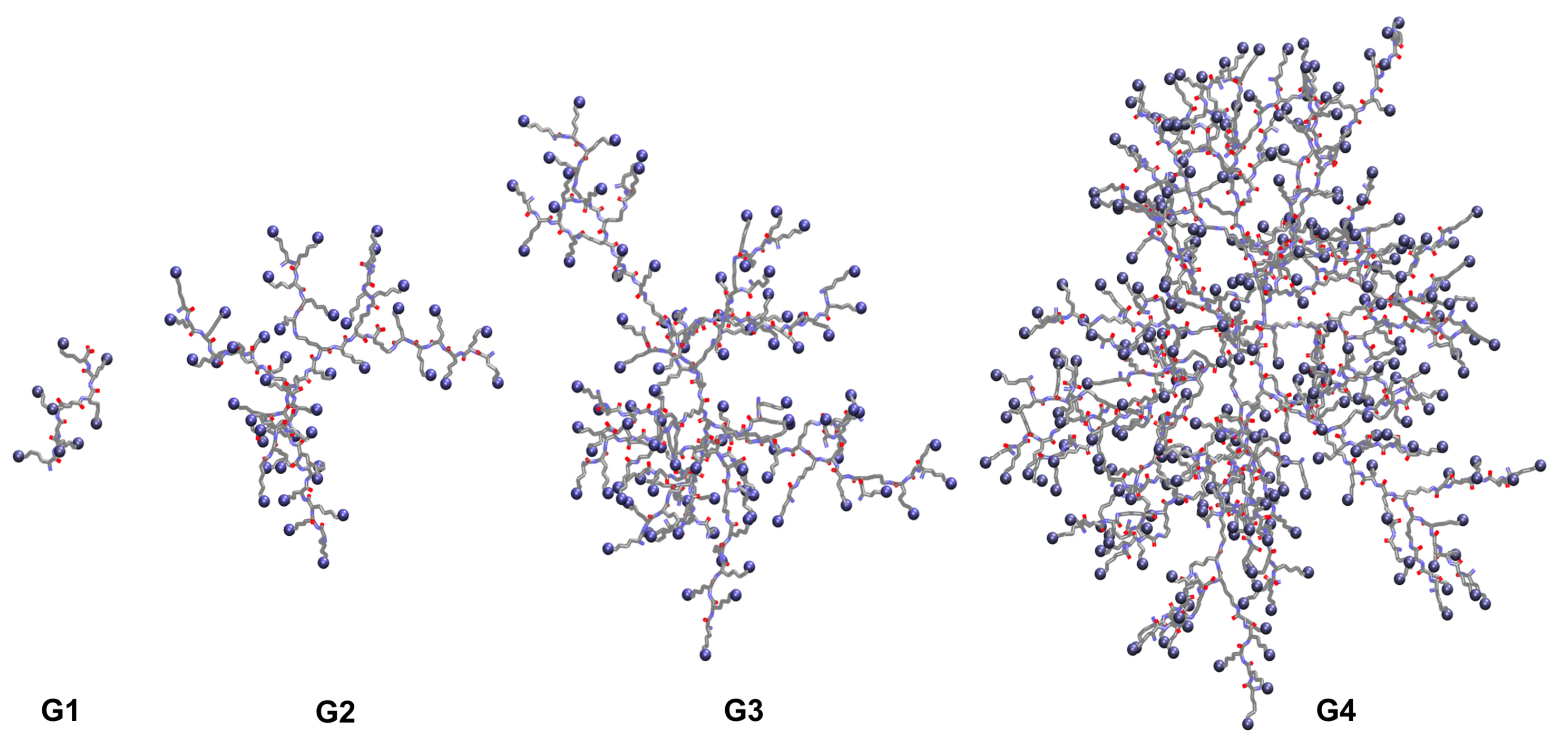

Figure 3: From left to right: snapshots of the first- to fourth-generation DGLs G1-G4 from microsecond MD simulations. Blue dots highlight the positively charged nitrogen atoms at physiological $\mathrm{pH}$. Adapted with permission from ref. [16], copyright: 2017 American Chemical Society.

\section{Functionalization and immobilization}

The presence of multiple amino groups on the arms, but also a free carboxylic acid function at the core of the native biomacromolecules, opens the door to various modifications toward the implementation of tailored features such as targeting, immobilization, fluorescence, or pharmacokinetic effects (Figure 4). The desired feature can be introduced either: i) directly (e.g. via a reductive amination for addressing DGLs with sugar moieties), if the chosen motif is adapted to coupling reactions that involve 
an amino group, or ii) through an intermediate linker (e.g. via a Michael addition for addressing DGLs with thiol-containing peptidic residues) if the chosen motif is unreactive in - or not compatible with - coupling reactions that involve an amino group. The linker may also carry non-innocent properties. For instance, a polyethylene glycol (PEG)-based linker may be of interest in order to extend the residence time in blood, reduce non-specific cellular uptake, or further lower the cytotoxicity of the nanoparticles. On the other hand, the core functionalization of DGLs involved the use of a prefunctionalized lysine building block that is introduced during the preparation of the first-generation dendrigraft. Contrary to the functionalization on their arms that leads to the simultaneous introduction of multiple motifs, the latter approach allows the introduction of a single motif on DGLs' skeleton.

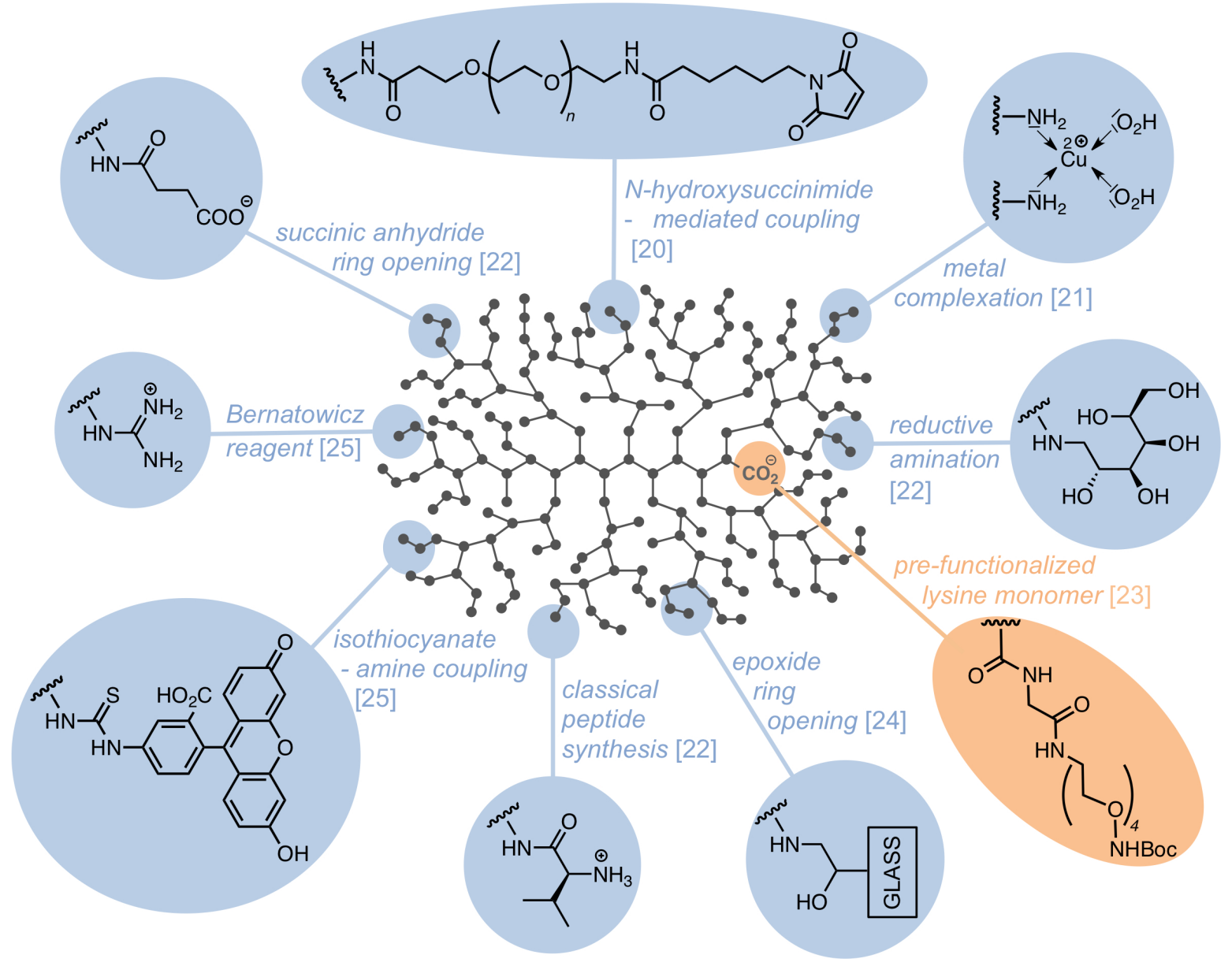

Figure 4: All reactions for arm and core modifications of the native DGLs reported in the literature so far. A unique example of introduced motif per type of reaction is showed here (with the corresponding references $[20,21,22,23,24,25])$.

The chemical modification of few terminal amine groups through the succinic anhydride motif led to efficient grafting of the third-generation DGL G3 onto plasma activated surfaces (i.e. PPE, polyimide, polystyrene, PTFE).[26] It turned out that the interactions of anionic ATP molecules on the surfaces were identical to that of the free DGL in solution in term of stoichiometry, showing that the immobilization procedure does not alter the binding properties of the dendritic polymer. Unmodified DLGs can also be attached on a solid support by using reactive polystyrene that bears epoxy functionalities.[27] 
For both approaches, AFM studies revealed that the resulting nanomaterials displayed monolayered homogeneous surfaces.

\section{Biology}

\section{Biocompatibility}

In order to enter the real world of industrial applications such as drug and gene delivery, biomaterial and tissue engineering, bioimaging or biosensing, polymers should display a number of crucial properties that we believe DGLs exhibit. Indeed, it was demonstrated that DGLs are readily soluble in water at least up to $60 \mathrm{~g} / \mathrm{L}$ in water,[12] are stable under sterilization conditions,[28] and are partially degradable under the action of endogenous peptidases.[29] In addition, these biomacromolecules are non-immunogenic[30] and carry low cytotoxicity (i.e. IC50 values on Hep2 cells of 16.8, 13.2, and $13.9 \mu \mathrm{g} . \mathrm{mL}^{-1}$ from second- to fourth-generation DGL, respectively),[31] in comparison with other polycationic polymers (e.g. about $90 \%$ viability for DGL G3 versus less than $50 \%$ viability for hyperbranched PEI at a same concentration of $12.5 \mu \mathrm{g} \cdot \mathrm{mL}^{-1}$ on HeLa cells).[32] As a result, DGLs already attracted the interest of multiple research groups during the last quinquennium, and found numerous applications in the biomedical field, where the ability of the arborescent polymers to display multiple functionalities has been exploited. Selected examples are presented below.

\section{Biocide}

Because polycationic polymers are known to possess inherent antibacterial activities that result from the binding to the microorganisms' cytoplasmic membrane and to their subsequent disruption, native DGLs have been considered as potential antibiotics. The interactions between DGLs and Gramnegative bacteria (i.e. Erwinia carotovora) were studied by Cottet et al. using capillary electrophoresis, with reported binding affinities in the micromolar range, and binding stoichiometries that decrease with increasing DGL generation (i.e. from $10.2 \times 10^{6}$ to $1.1 \times 10^{6}$ for DGL G2 to G4, respectively).[33] Moreover, a fast bacteriolytic activity of the second-generation DGL G2 was observed against Erwinia carotovora under low salt conditions.[34] This observation is interesting since Gram-negative bacteria due to a largely impermeable cell wall - are more resistant to antibiotics than Gram-positive bacteria. Although it is obvious that native DGLs display a small therapeutic window with respect to their antibacterial activity/cytotoxicity balance, one can now envisioned chemical modifications of DGLs, such as for example the quaternization of the free nitrogen atoms or the reduction of the charge density via the introduction of PEG chains, in order to improve both their biocide properties against Gram-negative bacteria and their cytocompatibility.[35]

\section{Drug and gene delivery}

Several obstacles such as fast degradation, low penetration through membranes, or low selectivity for target cells, often hinder the efficient delivery of bioactive molecules, including peptides or proteins. Sideratou et al. reported the efficient complexation of insulin with native and guanidine end-functionalized DGL G2, through electrostatic interactions between the partners at physiological pH.[36] The protection against enzymatic degradation as well as the retardation of insulin release in simulated intestinal fluid, which were found to be positively related to the number of guanidine end-groups, open interesting perspectives in the delivery of insulin to systemic circulation through oral administration. A therapeutic drug can also be covalently attached to a cationic dendrigraft, to obtain small-sized nanoparticles with strong penetrability in cells and tissues. However, small-sized nanoparticles are hampered by their fast systemic clearance and limited tumour retention. To address this issue, Gao et al. decorated a third-generation DGL G3 with multiple anticancer doxorubicin (Dox) motifs, was itself conjugated on the surface of large-sized gelatin nanoparticles.[37] Once in tumour 
microenvironments, high concentrations of the gelatin-degradating enzyme MMP-2 induced a shrinking event towards small-sized DGL-Dox conjugates. Such a multistage approach permits therefore to take advantage of both the enhanced retention properties of large-sized nanoparticles, and the tumour penetration of small-sized nanoparticles. To further improve the tumour targeting, an additional arginine-glycine-aspartic acid ligand, which is well-known to very efficiently bind the overexpressed $\alpha_{v} \beta_{3}$ integrin receptors on neo-vessel endothelial cells, can also be introduced on the nanotherapeutics' surface.[38]

In contrast to drug-based medicine, gene therapy targets the molecular cause of a disease instead of healing its consequences. It was demonstrated that positively charged amino groups of DGLs can interact with negatively charged phosphates of nucleic acids to yield polyplexes that protect the genetic material against DNAse I attack, and promote its cellular uptake via endocytosis or endocytosislike mechanism.[31] Regarding their plasmid DNA (pDNA) and silencing RNA (siRNA) internalizing properties in Hep2 cells, DGLs can be ranked as follows: G3 $>$ G4 $\geq \mathbf{G 2}$, with a higher effectiveness than the commercial transfection reagent Lipofectamine 2000. However, unmodified DGLs displayed disappointing transfection capabilities, which may results from their cytotoxicity at the high concentrations required in nucleic acid delivery experiments. Gene expression could be significantly boosted by the introduction of PEG chains on the dendrigrafts. For both DGLs G2 and G3, an increase in the covalent PEGylation extend resulted in an increase (i.e. up to four times) in gene pEGFP-N2 expression in HEK 293 cells, as well as a decrease of the polyplexes' cytotoxicity (e.g. at $200 \mu \mathrm{g} \cdot \mathrm{mL}^{-1}$, from $83 \pm 3 \%$ to $92 \pm 7 \%$ cell viability with a second-generation native DGL and a second-generation DGL-PEG, respectively).[39] These PEG chains can also be further labeled with known ligands for membrane proteins, thereby rendering gene vectors with targeting properties. For example, PEGylated DGL G3 was decorated by Jiang et al. (who are certainly the most active research group in the field of drug/gene/probe delivery using DGLs - A/N) with the short peptide EPRNEEK that is known to bind laminin receptors expressed on the blood-brain barrier and on cerebral glial cancer cells.[40] The resulting nanoparticles DGL-PEG-EPRNEEK were able to deliver in vivo a siRNA against survivin, which is an apoptosis inhibitor protein. In glioma-bearing mice, the untreated groups exhibited an early death as a function of time (i.e. $50 \%$ survival at 34 days), while DGLs-PEG/survivin groups showed no prolonged survival time, which was only increased in G3-PEG-EPRNEEK/survivin groups (i.e. $50 \%$ survival at 41 days). However, the expression of target receptors on normal cells and tissues is inevitable, which may lead to side effects of the nanoparticles. Some intrinsic characteristics of the tumour microenvironment can be exploited towards enhanced tumour/background accumulation ratio. Jiang et al. showed that the variation of the extracellular $\mathrm{pH}$ could be used to activate/inactivate a receptor-mediated endocytosis by conjugating the shielding molecule diethylenetriaminepentaaceticacid (DTPA) to the transferin membrane protein-targeting peptide HAIYPRH via a $\mathrm{pH}$-sensitive hydrazone bond.[41] Under normal conditions ( $\mathrm{pH}$ 7.4), the resulting G3-PEGHAIYPRH-hydrazone-DTPA nanoparticle was not able to deliver a pDNA encoding for the green fluorescent protein, whereas the nanoparticle was considerably absorbed in tumor acidic environment ( $\mathrm{pH}$ 6.5). In addition, the non-hydrolysable G3-PEG-HAIYPRH-DTPA/pDNA ensemble was inactive against transferin-expressing cells in acidic conditions, demonstrating therefore the $\mathrm{pH}$-activated detachment of the shielding molecule.

Then, combining gene and drug delivery may be regarded as a potential strategy for achieving enhanced therapeutic activities. Using the previously described vector G3-PEG-HAIYPRH, it has been shown that Dox as a chemotherapeutic drug to kill tumor cells, and anti-VEGF (i.e. vascular endothelial growth factor) as a gene to inhibit tumor angiogenesis, both synergically promoted a remarkable extend of median survival time in treated glioma-bearing mice (i.e. twice as long in comparison untreated animals).[42] Another level of sophistication can be added to the former nanoparticle approach by replacing the targeting ligand by an activatable cell-penetrating peptide that is dual-triggered by the pH and MMP-2.[43] The resulting nanotherapeutics displayed a superior efficiency against glioma as a result of both tumor targeting and internalization functions. This last example certainly describes 
one of the most elaborated DGL-based nanotherapeutics to date.

\section{Imaging}

Early detection and monitoring is essential to the successful treatment of many diseases including cancer and neurodegenerative diseases. Therefore, imaging agents should display high affinity, selectivity for targeted tissues or cells. Various strategies involving DGLs have been reported in the literature towards bioimaging:

- The coupling through a PEG linker of a conventional Gd(III)-pentetic acid complex. When associated with a neuroectodermal tumour-specific ligand (i.e. chlorotoxin), the precise detection of glioma located behind the blood-brain barrier was possible by magnetic resonance imaging.[44]

- The delivery of a green fluorescent protein-encoding plasmid. When associated with a peptidic ligand for the low-density lipoprotein receptor-related protein (i.e. angiopep), the nanoparticles permitted the visualisation by fluorescence microscopy of SH-SY5Y cells, which are a model of dopaminergic neurons in Parkinson's disease.[45] At the same concentration, the DGL-based nanoparticles showed higher cellular uptake and gene expression compared to the PAMAM-based equivalent. In addition, they exhibited no apparent cytotoxicity against the cell line.

- The non-covalent absorption of DGLs on superparamagnetic iron oxide nanoparticles. When associated with a peptidic ligand for $\alpha_{v} \beta_{3}$ integrin receptors (i.e. RGD), the nanoparticles permitted in vivo magnetic resonance imaging of living mice bearing HepG2 tumors.[46]

- The coupling with an organic fluorophore (i.e. Bodipy) through a hydroxysuccinimide-amine reaction for the 3D localization of DGL/DNA complexes in mice by CT scanning.[47] In some cases, the optical signal may also be turned on by desired events, such as a $\mathrm{pH}$-dependant electron transfer between the fluorophore and a quencher,[48] or the separation of the fluorophore and a quencher upon enzymatic cleavage.[49] Such approaches minimize the background signal for increased specificity and sensitivity of the images.

In most cases, the probes were associated with oligonucleotides and/or drugs for a concomitant diagnostic and therapeutic action. In a very recent example, Guo et al. decorated a third-generation DGL with two aptamers (i.e. a nucleolin-specific binding aptamer, AS1411, and a cytochrome c aptamer) for targeting, and a fluorophore (i.e. Cy 5.5) for imaging. Dox was intercalated into a DNA duplex containing an ATP aptamer, which was subsequently condensed by DGL to form a theragnostic

nanoparticle (Figure 5).[50] This elegant system has demonstrated to not only selectively accumulate in the mitochondria of HeLa cancer cells, but also to generate a distinct near-infrared fluorescence signal in the tumor region and to promptly release the loaded Dox in virtue of the high concentrations of ATP in mitochondria. 

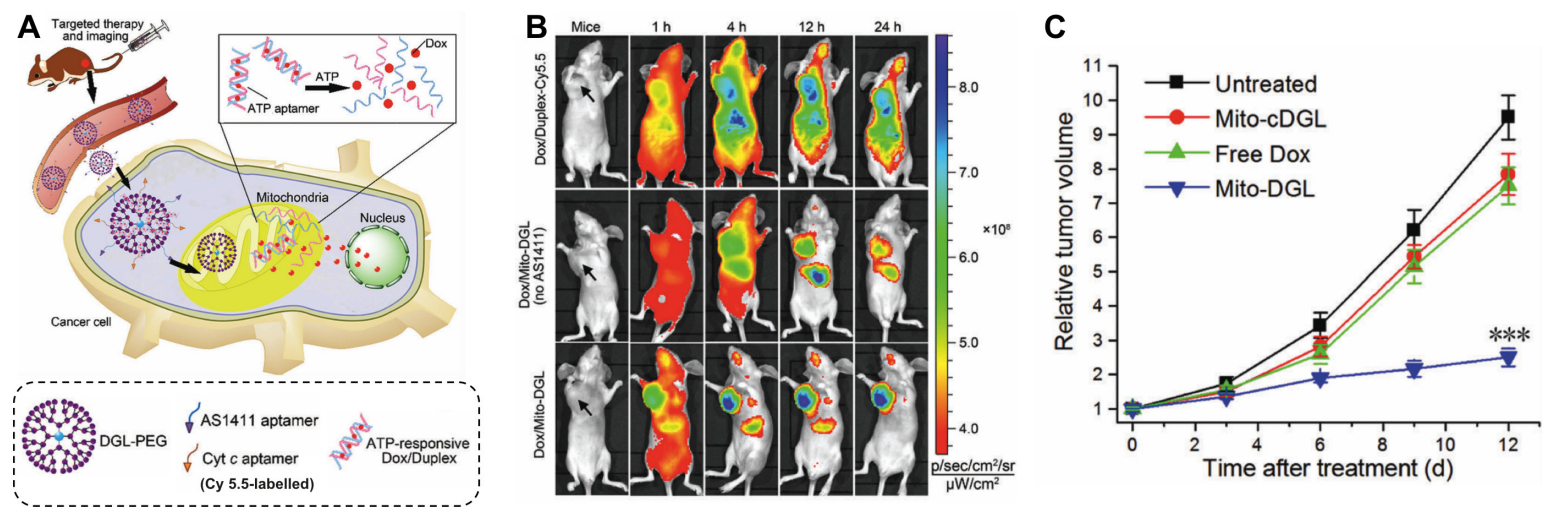

Figure 5: (A) Schematic illustration of the G3-based theragnostic nanoparticle (Dox/Mito-DGL) structure, and the mechanism of mitochondria-targeting drug release triggered by ATP; $(B)$ timedependent in vivo fluorescence images of subcutaneous HeLa tumor-bearing mice after i.v. injection of Dox/duplex-Cy5.5, Dox/Mito-DGL (no AS1411) or Dox/Mito-DGL; $(C)$ change in the relative tumor volume $(\mathrm{V} / \mathrm{V} 0)$ upon different treatments (Dox/Mito-cDGL is obtained by replacing the duplex in Dox/Mito-DGL by a non ATP-responsive duplex). Data are means $\pm \mathrm{SD}(\mathrm{n}=6),{ }^{* * *} \mathrm{P}<0.001$ compared to other groups using a one-way ANOVA. Adapted with permission from ref. [50], copyright: 2017 Royal Society of Chemistry.

\section{Tissue engineering}

Tissue engineering aims at the regeneration of damaged or dysfunctional tissues ands organs through the use of polymeric scaffolds that promote cell adhesion and/or deliver growth factors. In the context of regenerative medicine for peripheral nerve, DGLs were identified to be superior to linear poly$L$-lysines of similar molecular weights in promoting nerve cell adhesion, suggesting that nerve cells adhered more efficiently to the dendritic-coated poly(glycolic acid) fibres.[51] In addition, DGL G4, in association with a poly ( $\epsilon$-caprolactone) membrane, was also an effective nanoreservoir for the nerve growth factor; the resulting biomaterial being able to induce the full vascularization and innervation of a tooth in vivo.[52]

\section{Biosensing}

In a biosensing purpose, a third-generation generation DGL G3, immobilized on plasma activated polypropylene filters, was reported to be an efficient capture agent for concentrating bacteriophage MS2, allowing the quantification of the model virus in large volumes of water.[53] This approach relies on the non-covalent interactions that take place between positively charged dendrigrafts and the negatively charged surface of the viral particles. It is interesting to note that, since DGLs and bacteria also displayed high affinities based on electrostatic interactions [33], this approach could be extended to the detection and/or removal of pathogenic bacteria from contaminated waters. Recently, Vial et al. envisioned that a DGL might be able to mimic the highly basic protamine, a heparin neutralizing protein. Heparin is a polysulfated glycosaminoglycan (GAG) that is extensively administered to patients as an intravenous anticoagulant, and its quantification in clinical settings is highly desirable in order to prevent thrombophilia or bleeding disorders. It turned out that DGLs were able to form multi-ligand complexes with a poly-anionic fluorescent peptide, leading to the decrease of the optical signal that can be restored upon the introduction of heparin, in a typical indicator-displacement regime (Figure 6).[54] This simple system allows - for the first time - the detection and the quantification of the anticoagulant in human blood at clinically relevant levels. In the same conditions and at comparable molecular weight, LPL was not able to bind the anionic probe, highlighting therefore the importance 
of the dendritic topology for a strong association event between the binding partners when working in complex media. Remarkably, the highly concentrated circulating protein serum albumin (i.e. about 40 grams per litre of blood), which was reported to display dissociation constants with DGLs in the micromolar range,[55] did not seem to interfere with the sensing process, therefore highlighting the tremendous affinity of dendrigrafts for heparin. Also, depending on the loading of the indicator on the receptor, various GAGs induced an increase or a decrease of the optical signal as they displace the indicators from the receptor or they compact the indicators on the receptor's surface, respectively.[56] This unique feature allowed the blind identification of various GAGs by linear discrimination analysis with a level of accuracy of $100 \%$.
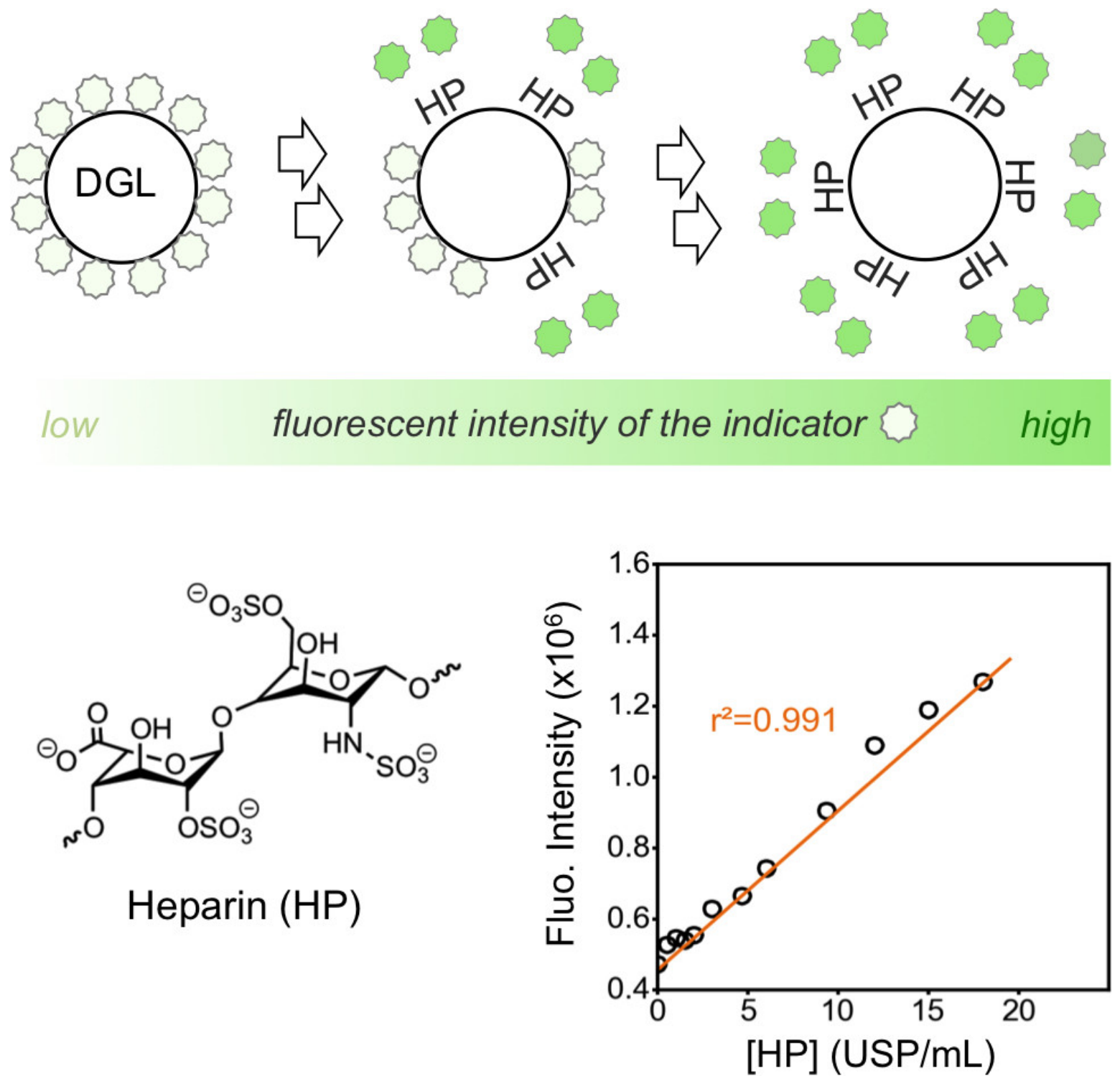

Figure 6: Schematic representation of the indicator-displacement assay using a DGL-fluorescent indicator complex (top), and application to the sensing of heparin (HP) from heparinized human blood samples (bottom). Adapted with permission from ref. [54], copyright: 2015 Royal Society of Chemistry. 


\section{Conclusion}

Here, we deliver a digest of the diverse research works that involved the newcoming poly- $L$-lysine dendrigrafts so far. The ambition of this minireview is a better exposure of these macromolecules, with the objective that they will eventually realize their full potential. Although DGLs have been reported in some biological studies to be superior to other linear or dendritic cationic polymers, their ability to actually respond to medical needs has still to be demonstrated. This includes clinical data, and imposes collaboration with physicians/medical researchers for potential clinical translations. In this biomedical context, the attractiveness of these macromolecules relies on their: (i) straightforward, robust and green synthesis on a multi-gram scale, (ii) strong positive electrostatic potential and conformational elasticity for highly efficient interactions with biologically relevant anionic species, (iii) chemical flexibility for further functionalization to implement desirable properties, and (iv) biodegradability, low toxicity and non-immunogenicity. Given these features and the success met so far by poly- $L$-lysine dendrigrafts, we believe that they are promising next-generation biopolymers for real-world applications.

\section{Acknowledgment}

This work was supported by the Centre National de la Recherche Scientifique (CNRS), the Université de Lyon, and the Université de Montpellier.

\section{Keywords}

Dendritic polymers, Poly- $L$-lysines, Non-viral vectors, Antibacterial agents, Material and Tissue engineering, Biosensing 
Frontispiece

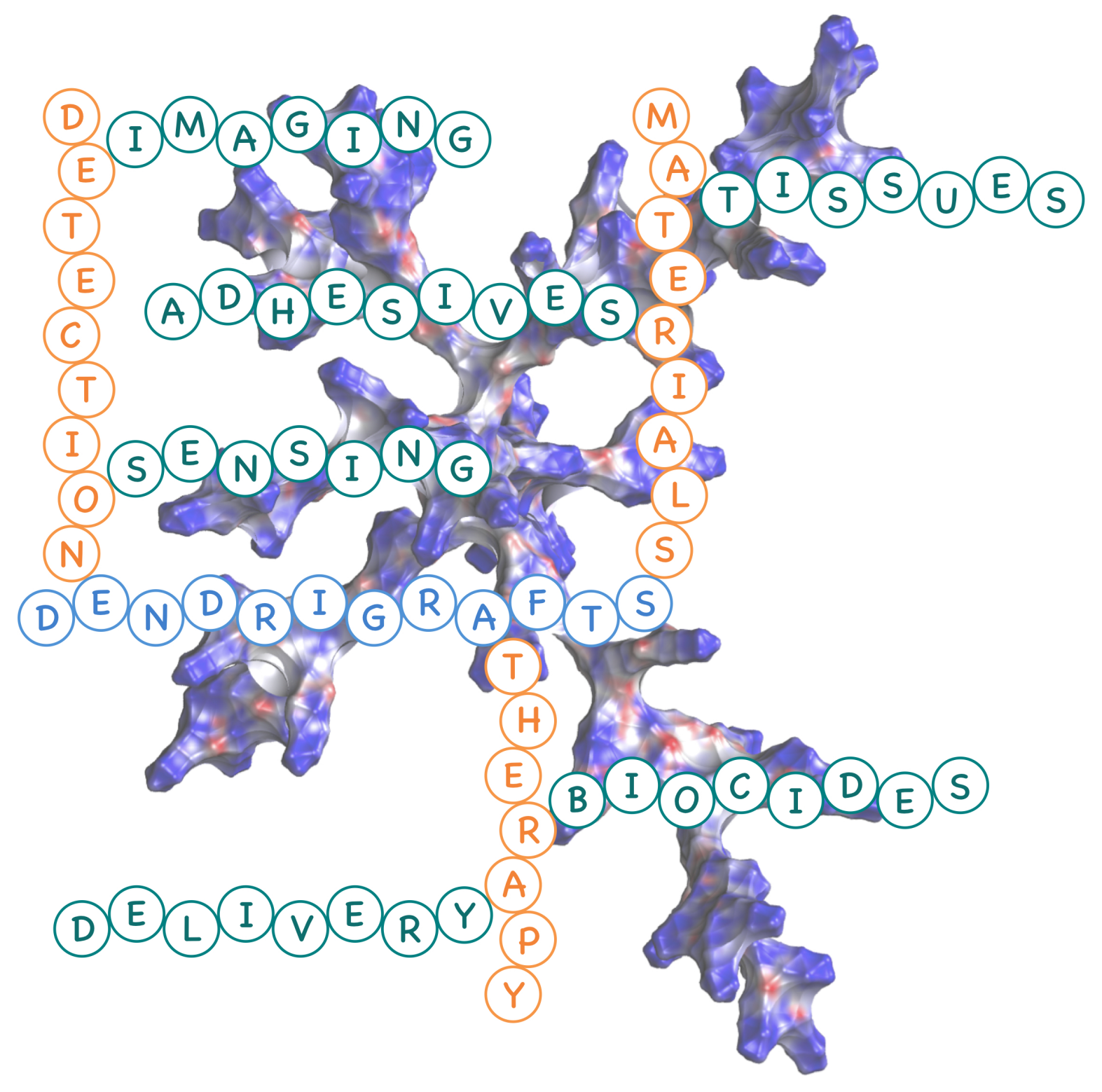

Figure 7: Frontispiece 


\section{Table of Content}

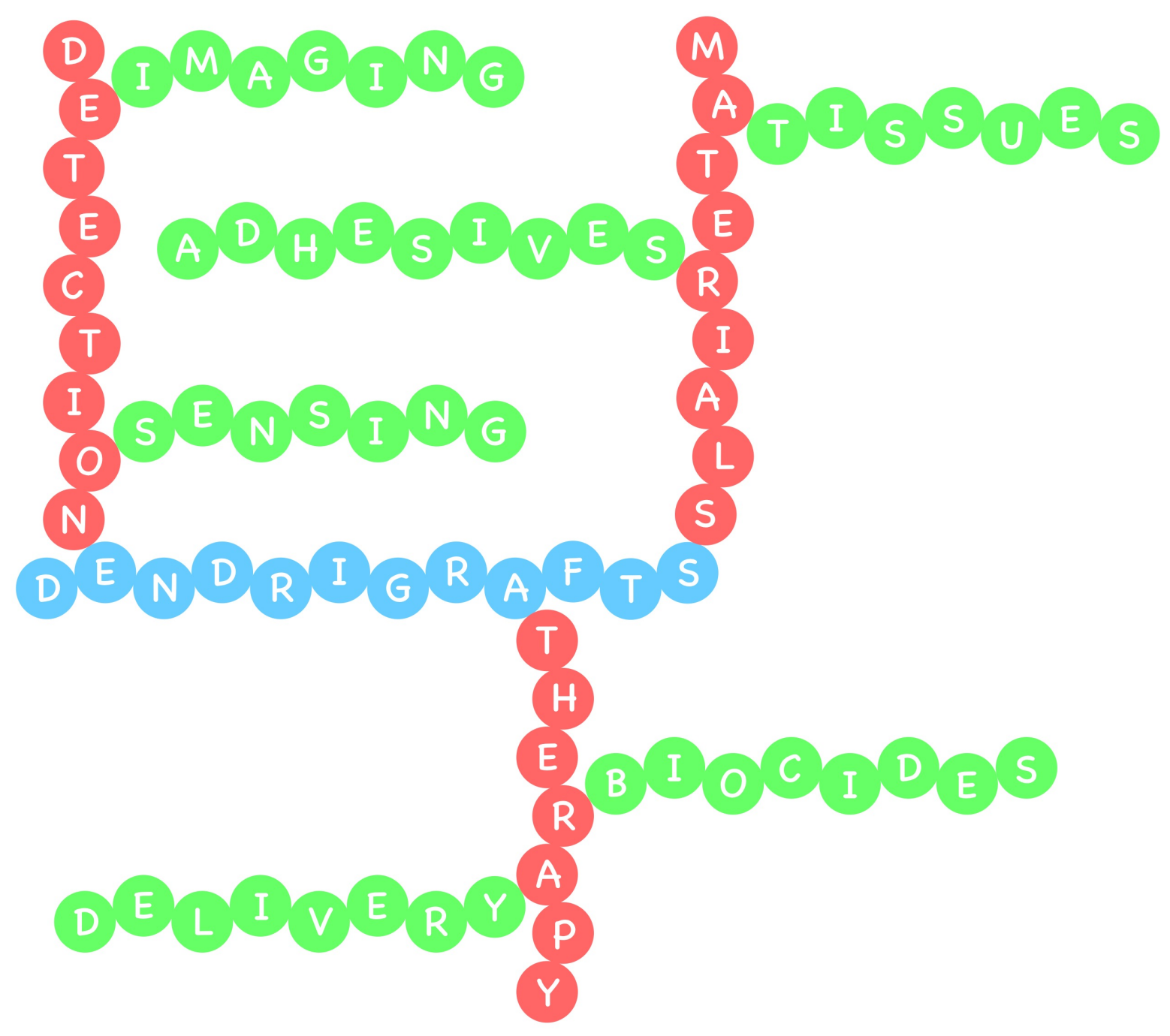

Figure 8: Graphical Abstract

Talkin' 'bout my generation: despite a growing interest for poly-L-lysine dendrigrafts, there is still plenty of room for further exiting developments that could result from a better exposure of these macromolecules, which is the ambition of this minireview. 


\section{Biographies}

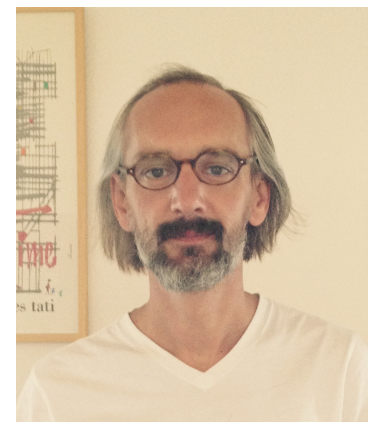

During his $\mathrm{PhD}$ and Post-doc, Laurent Vial was lucky enough to work under the guidance of two awesome supervisors: Prof. Jérôme Lacour (University of Geneva, Switzerland) and Prof. Jeremy Sanders (University of Cambridge, UK), respectively. He is a CNRS research scientist (University of Lyon, France), currently working on artificial receptors for biologically relevant molecules with applications as biosensors or therapeutics. At an early age, Laurent showed tremendous aptitude for being right all of the time. He constantly puts this skill to work on Jean-Patrick Francoia.

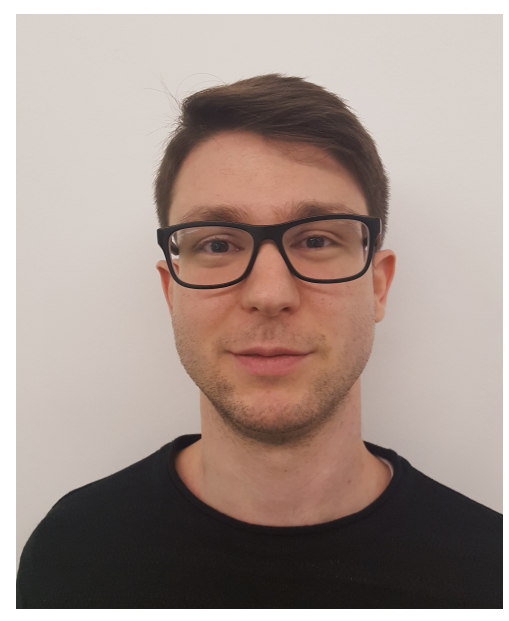

Jean-Patrick Francoia was born in France in 1990. He obtained his master degree from the University of Nice Sophia Antipolis, after a year as an Erasmus student in Prof. Kenneth Wörnmark's group (Lund, Sweden). In 2016, he received his Ph.D. degree in chemistry from the University of Montpellier (France), exploring the use of poly- $L$-lysine dendrigrafts as biosensors under the supervision of Dr. Laurent Vial. Since March 2017, he has been a Postdoctoral Research Assistant in the laboratory of Prof. Leroy Cronin at the University of Glasgow (Scotland). His current research interests include the development of 3D printed architectures for embedded organic synthesis and the digitization of chemistry.

\section{References}

[1] D. A. Tomalia, Mater. Today 2005, 8, 34-46.

[2] S. J. Teertstra, M. Gauthier, Prog. Polym. Sci. 2004, 29, 277-327. 
[3] R. G. Denkewalter, J. Kolc, W. J. Lukasavage, US Pat. 4289872, 1981.

[4] D. A. Tomalia, H. Baker, J. Dewald, M. Hall, G. Kallos, S. Martin, J. Roeck, J. Ryder, P. Smith, Polym. J. 1985, 17, 117-132.

[5] J. P. Tam, Proc. Natl. Acad. Sci. USA 1988, 85, 5409-5413.

[6] S. K. Samal, M. Dash, S. Van Vlierberghe, D. L. Kaplan, E. Chiellini, C. van Blitterswijk, L. Moroni, P. Dubruel, Chem. Soc. Rev. 2012, 41, 7147-7194.

[7] M. Labieniec-Watala, C. Watala, J. Pharm. Sci. 2015, 104, 2-14.

[8] Q. Mou, Y. Ma, X. Jin, X. Zhu, Mol. Syst. Des. Eng. 2016, 1, 25-39.

[9] Z. Kadlecova, L. Baldi, D. Hacker, F. M. Wurm, H. A. Klok, Biomacromolecules 2012, 13, 31273137.

[10] M. Scholl, T. Q. Ngyen, B. Bruchmann, H.-A. Klok, J. Polym. Sci. Pol. Chem. 2007, 45, 54945508 .

[11] S. Svenson, Chem. Soc. Rev. 2015, 44, 4131-4144.

[12] H. Collet, E. Souaid, H. Cottet, A. Deratani, L. Boiteau, G. Dessalces, J.-C. Rossi, A. Commeyras, R. Pascal, Chem. Eur. J. 2010, 16, 2309-2316.

[13] H. Cottet, M. Martin, A. Papillaud, E. Souaïd, H. Collet, A. Commeyras, Biomacromolecules 2007, 8, 3235-3243.

[14] B. Maret, A. Crépet, C. Faye, L. Garrelly, C. Ladavière, Macromol. Chem. Phys. 2015, 216, 95-105.

[15] A. Ibrahim, D. Koval, V. Kašička, C. Faye, H. Cottet, Macromolecules 2013, 46, 533-540.

[16] J.-P. Francoia, J.-C. Rossi, G. Monard, L. Vial, J. Chem. Inf. Model. 2017, 57, 2173-2180.

[17] G. Coussot, E. Nicol, A. Commeyras, I. Desvignes, R. Pascal, O. Vandenabeele-Trambouze, Polym. Int. 2009, 58, 511-518.

[18] G. Herzog, S. Flynn, C. Johnson, D. W. M. Arrigan, Anal. Chem. 2012, 84, 5693-5699.

[19] C. Johannessen, J. Kapitán, H. Collet, A. Commeyras, L. Hecht, L. D. Barron, Biomacromolecules 2009, 10, 1662-1664.

[20] S. An, Y. Kuang, T. Shen, J. Li, H. Ma, Y. Guo, X. He, C. Jiang, Biomaterials 2013, 34, 8949-8959.

[21] J. C. Rossi, B. Maret, K. Vidot, J. P. Francoia, M. Cangiotti, S. Lucchi, C. Coppola, M. F. Ottaviani, Macromol. Biosci. 2015, 15, 275-290.

[22] J.-C. Rossi, L. Boiteau, H. Collet, B. Mbondo Tsamba, N. Larcher, R. Pascal, Tetrahedron Lett. 2012, 53, 2976-2979.

[23] A. M. Bondia, N. Larcher, L. Garrelly, J. C. Rossi, R. Pascal, Tetrahedron Lett. 2010, 51, 33303333.

[24] G. Coussot, C. Faye, A. Ibrahim, M. Ramonda, M. Dobrijevic, a. Le Postollec, F. Granier, O. Vandenabeele-Trambouze, Anal. Bioanal. Chem. 2011, 399, 2295-2302.

[25] I. Tsogas, T. Theodossiou, Z. Sideratou, C. M. Paleos, H. Collet, J. C. Rossi, B. Romestand, A. Commeyras, Biomacromolecules 2007, 8, 3263-3270. 
[26] B. Couturaud, A. M. Bondia, C. Faye, L. Garrelly, A. Mas, J. J. Robin, J. Colloid Interface Sci. 2013, 408, 242-251.

[27] C. Lorion, C. Faye, B. Maret, T. Trimaille, T. Régnier, P. Sommer, R. Debret, J. Biomater. Sci. Polym. Ed. 2014, 25, 136-149.

[28] G. Coussot, T. Moreau, C. Faye, F. Vigier, M. Baqué, A. Le Postollec, S. Incerti, M. Dobrijevic, O. Vandenabeele-Trambouze, Int. J. Astrobiol. 2016, 2, 1-10.

[29] J. Chamieh, J. P. Biron, L. Cipelletti, H. Cottet, Biomacromolecules 2015, 16, 3945-3951.

[30] B. Romestand, J.-l. Rolland, A. Commeyras, I. Desvignes, R. Pascal, O. Vandenabeele-trambouze, Biomacromolecules 2010, 11, 1169-1173.

[31] J. Hofman, M. Buncek, R. Haluza, L. Streinz, M. Ledvina, P. Cigler, Macromol. Biosci. 2013, 13, 167-176.

[32] M. Tang, H. Dong, Y. Li, T. Ren, J. Mater. Chem. B 2016, 4, 1284-1295.

[33] N. Sisavath, P. Got, G. M. Charrière, D. Destoumieux-Garzon, H. Cottet, Anal. Chem. 2015, 87, 6761-6768.

[34] F. Oukacine, B. Romestand, D. M. Goodall, G. Massiera, L. Garrelly, H. Cottet, Anal. Chem. 2012, 84, 3302-3310.

[35] Y. Xue, H. Xiao, Y. Zhang, Int. J. Mol. Sci. 2015, 16, 3626-3655.

[36] Z. Sideratou, N. Sterioti, D. Tsiourvas, L. A. Tziveleka, A. Thanassoulas, G. Nounesis, C. M. Paleos, J. Colloid Interface Sci. 2010, 351, 433-441.

[37] G. Hu, Y. Wang, Q. He, H. Gao, RSC Adv. 2015, 5, 85933-85937.

[38] G. Hu, H. Zhang, L. Zhang, S. Ruan, Q. He, H. Gao, Int. J. Pharm. 2015, 496, 1057-1068.

[39] R. Huang, S. Liu, K. Shao, L. Han, W. Ke, Y. Liu, J. Li, S. Huang, C. Jiang, Nanotechnology 2010, 21, 265101.

[40] Y. Liu, X. He, Y. Kuang, S. An, C. Wang, Y. Guo, H. Ma, J. Lou, C. Jiang, Mol. Pharm. 2014, 11, 3330-3341.

[41] L. Han, Y. Guo, H. Ma, X. He, Y. Kuang, N. Zhang, E. Lim, W. Zhou, C. Jiang, Small 2013, 9, $3647-3658$.

[42] Y. Kuang, X. Jiang, Y. Zhang, Y. Lu, H. Ma, Y. Guo, Y. Zhang, S. An, J. Li, L. Liu, Y. Wu, J. Liang, C. Jiang, Mol. Pharm. 2016, 13, 1599-1607.

[43] S. Huang, K. Shao, Y. Liu, Y. Kuang, J. Li, S. An, Y. Guo, C. Jiang, ACS Nano 2013, 7, $2860-2871$.

[44] R. Huang, L. Han, J. Li, S. Liu, K. Shao, Y. Kuang, X. Hu, X. Wang, H. Lei, C. Jiang, Biomaterials 2011, 32, 5177-5186.

[45] R. Huang, H. Ma, Y. Guo, S. Liu, Y. Kuang, K. Shao, J. Li, Y. Liu, L. Han, S. Huang, S. An, L. Ye, J. Lou, C. Jiang, Pharm. Res. 2013, 30, 2549-2559.

[46] J.-M. Shen, X.-X. Li, L.-L. Fan, X. Zhou, J.-M. Han, M.-K. Jia, L.-F. Wu, X.-X. Zhang, J. Chen, Int. J. Nanomed. 2017, 12, 1183-1200.

[47] Y. Liu, Y. Guo, S. An, Y. Kuang, X. He, H. Ma, J. Li, J. Lu, J. Lv, N. Zhang, C. Jiang, PloS one 2013, 8, e62905. 
[48] L. Han, M. Liu, D. Ye, N. Zhang, E. Lim, J. Lu, C. Jiang, Biomaterials 2014, 35, 2952-2960.

[49] Y. Liu, Y. Hu, Y. Guo, H. Ma, J. Li, C. Jiang, J. Control. Release 2012, 163, 203-210.

[50] H. Chen, J. Tian, D. Liu, W. He, Z. Guo, J. Mater. Chem. B 2017, 5, 972-979.

[51] C. Kojima, E. Fusaoka-Nishioka, T. Imai, A. Nakahira, H. Onodera, J. Biomed. Mater. Res. Part A 2016, 104, 2744-2750.

[52] S. Eap, T. Bécavin, L. Keller, T. Kökten, F. Fioretti, J. L. Weickert, E. Deveaux, N. BenkiraneJessel, S. Kuchler-Bopp, Adv. Healthc. Mater. 2014, 3, 386-391.

[53] A. Cadiere, B. Couturaud, J. Boismard, P. Le Cann, A. Gérard, A. Mas, C. Faye, L. Garrelly, B. Roig, J. Appl. Microbiol. 2013, 115, 290-297.

[54] J.-P. Francoia, R. Pascal, L. Vial, Chem. Commun. 2015, 51, 1953-1956.

[55] N. Sisavath, L. Leclercq, T. Le Saux, F. Oukacine, H. Cottet, J. Chrom. A 2013, 1289, 127-132.

[56] J.-P. Francoia, L. Vial, Chem. Commun. 2015, 51, 17544-17547. 